\title{
Reacciones post vacuna SINOPHARM COVID-19 en el personal de salud -Huancayo 2021.
}

\author{
Post vaccine reactions SINOPHARM COVID-19 in health personnel -Huancayo 2021
}

\author{
Jenny Giovanna Poma Salinas ${ }^{1, a}$, Belinda Olga Garcia Inga ${ }^{1, b}$, Marivel Rosa Martínez Véliz ${ }^{1, c}$, Rosario \\ Eleana Cuadros Ríos ${ }^{1, d}$
}

\section{RESUMEN}

En un esfuerzo mundial por combatir la pandemia de COVID-19, se inicia la vacunación contra la COVID-19, en el Perú el 9 de febrero 2021, vacunando prioritariamente al personal de salud con la vacuna de SINOPHARM. Siendo desconocido lo que esta vacuna traería consigo como reacciones, surge el presente trabajo. Objetivo: Identificar las reacciones más frecuentes post vacuna contra el Covid-19 SINOPHARM en la primera y segunda dosis en el personal de Salud del Hospital El Carmen, Huancayo 2021. Material y Métodos: Estudio básico y descriptivo, la muestra conformada 284 miembros del personal del hospital. Resultados: El 71,1\% tiene de 20 a 49 años, $80,3 \%$ son de sexo femenino, 33,4\% reaccionaron en la primera dosis, 48,9\% reaccionaron en la segunda dosis, 59,2\% salió positivo a COVID 19 antes de ser vacunado, 18,3\% salió positivo después de ser vacunado contra la COVID 19, 39,4\% no tuvieron ninguna reacción inmediata en la primera dosis, 18,3\% tuvieron reacciones locales: $16,2 \%$ hinchazón, 16,2\% picor en la zona de vacunación, 4,9\% escleroderma , 4,9\% salpullido; en la segunda dosis, el $62,7 \%$ no tuvo ninguna reacción, 10,9\% hinchazón, 9,5\% picor en la zona de vacunación, 7\% reacciones locales, $7 \%$ salpullido, 2,8\% escleroderma, 54,5\% en la primera dosis, 90,1\% no reaccionaron posteriormente en la segunda dosis. Conclusiones: las reacciones más frecuentes post vacuna contra el Covid-19 SINOPHARM en la primera y segunda dosis en el personal de Salud del Hospital, fueron dolor muscular $16,6 \%$ en la primera dosis y $13,5 \%$ en la segunda dosis.

PALABRAS CLAVES: Covid 19, vacuna, sinopharm, reacciones adversas, paciente, unidad de cuidados intensivos, perfil epidemiológico.

\section{SUMMARY}

In a global effort to combat the COVID-19 pandemic, vaccination against COVID-19 begins in Peru on February 9, 2021, with priority vaccinating health personnel with the SINOPHARM vaccine. Being unknown what this vaccine would bring with it as reactions, the present work arises. Objective: To identify the most frequent reactions after the vaccine against Covid-19 SINOPHARM in the first and second doses in the Health personnel of Hospital El Carmen, Huancayo 2021. Material and Methods: Basic and descriptive study, the sample made up 284 hospital personnel. Results: $71.1 \%$ are between 20 and 49 years old, $80.3 \%$ female, $33.4 \%$ reacted in the first dose, $48.9 \%$

\footnotetext{
Universidad Privada de Huancayo Franklin Roosevelt Huancayo. Huancayo, Perú.

Docente; Lic. Enfermería; ORCID ID: 0000-0002-3325-1258

Docente; Lic. Enfermería; Magister ; ORCID ID: 0000-0003-1500-5253

Docente; Lic. Enfermería; Magister ; ORCID ID: 0000-0003-1992-1865

Docente; Lic. Enfermería; Magister ; ORCID ID: 0000-0003-1996-1621
} 
reacted in the second dose, $59.2 \%$ tested positive for COVID 19 before being vaccinated, $18.3 \%$ tested positive after being vaccinated against COVID 19, 39.4\% had no immediate reaction in the first dose, $18.3 \%$ had local reactions, $16.2 \%$ swelling, $16.2 \%$ itching in the vaccination area, $4.9 \%$ scleroderma, $4.9 \%$ rash; $62.7 \%$ had no reaction in the second dose, $10.9 \%$ swelling, $9.5 \%$ itching in the vaccination area, $7 \%$ local reactions, $7 \%$ rash, $2.8 \%$ scleroderma, $54.5 \%$ in the first dose, $90.1 \%$ did not react later in the second dose. Conclusions: the most frequent post-vaccine reactions against Covid-19 SINOPHARM in the first and second doses in the Hospital Health personnel were muscle pain, $16.6 \%$ in the first dose and $13.5 \%$ in the second dose.

KEYWORDS: Covid 19, vaccine, sinopharm, adverse reactions.

\section{INTRODUCCIÓN}

La investigación titulada: reacciones más frecuentes post vacuna contra la covid-19 para la primera y segunda dosis en el personal de salud del Hospital "El Carmen" Huancayo 2021. Se originó en el interés de aportar datos importantes sobre la vacunación con Sinopharm en pandemia que aqueja al mundo por que la OMS y sus asociados trabajan juntos en la respuesta, siguiendo el rastro de la pandemia, ofreciendo asesoramiento sobre las intervenciones más importantes, distribuyendo suministros médicos esenciales a los más necesitados y se han lanzado a la carrera por encontrar una vacuna. Al 18 de febrero de 2021, hay al menos siete vacunas distintas que los países han empezado a administrar en tres plataformas, concediendo prioridad en todos los casos a las personas vulnerables (1).

La vacuna desarrollada por la farmacéutica estatal china Sinopharm y el Instituto de Productos Biológicos de Pekín, luego de culminar la última fase de ensayos clínicos nos refiere que dicha vacuna cuenta con una eficacia del $79 \%$. Se conoce que la vacuna en su etapa de prueba fue inyectada a voluntarios en la Universidad Peruana Cayetano Heredia en Lima, Perú (2).

Reacciones locales: enrojecimiento, hinchazón, escleroma, sarpullido, picor en el lugar de la aplicación de la vacuna; y para las reacciones sistémicas: Dolor de cabeza, fiebre, fatiga, dolor muscular, dolor de articulaciones, tos, dificultad para respirar, náuseas, diarreas, picor de la piel, mareos, pérdida del apetito, vómitos, dolor orofaríngeo, dificultad para tragar, goteo nasal, estreñimiento, hipersensibilidad, letargo, somnolencia, dificultad para dormir, estornudos, nasofaringitis, congestión nasal, sequedad de la garganta, gripe, hipoestesia, dolor de extremidades, palpitaciones, dolor de estómago, sarpullido, anomalías de la piel, anomalía de las membranas mucosas, acné, dolor de ojos, molestias en los oídos, linfadenopatía, escalofríos, disgeusia (distorsión del sentido del gusto), pérdida del gusto, sensación anormal, temblores, dificultad para prestar atención, hemorragias nasales, asma, irritación de la garganta, amigdalitis, molestias en las extremidades, dolor de cuello, dolor de mandíbula, bultos en el cuello, úlceras en la boca, dolor de muelas, enfermedad esofágica, gastritis, decoloración de las heces, dolor de ojos, visión borrosa, irritación de los ojos, pérdida de visión (ceguera), dolor de oídos, nerviosismo, alta presión sanguínea, baja presión sanguínea, incontinencia urinaria, y retraso de la menstruación (3).

La reacción adversa más frecuente fue el dolor en el sitio de inoculación, con una frecuencia de $42,1 \%$ y $43,2 \%$ en la primera y segunda dosis. Las reacciones sistémicas más frecuentes fueron cefalea y astenia. No se reportaron reacciones adversas graves. La presencia de enfermedades crónicas representó un mayor riesgo de tener reacciones adversas ( $<<0,05, \mathrm{OR}=3,6, \mathrm{IC} 95 \%$ 1,21-10,7) además el antecedente alergias conocidas presentó una asociación estadísticamente significativa $(\mathrm{x} 2=5,1, \mathrm{p}<0,05)$ Las reacciones adversas a la primera dosis aumentaban la probabilidad de ocurrencia de estas en una segunda dosis $(\mathrm{p}<0,001, \mathrm{OR}=4,71, \mathrm{IC} 95 \% 1,92$ 11,5). La frecuencia de reacciones adversas en general fue de $75,8 \%$. Los factores asociados fueron el haber presentado el antecedente de enfermedades crónicas y tener alguna alergia conocida a medicamentos y/o alimentos, además el haber presentado alguna reacción adversa en la primera dosis aumentaba el riesgo de presentarlas en la segunda (5).

Los efectos adversos fueron de gravedad leve o moderada y se desarrollaron durante los primeros siete días después de la inoculación. Los efectos adversos locales fueron los siguientes: dolor en el sitio de la inyección (>10\%); eritema, hiperemia, prurito, edema o hinchazón en el sitio de la inyección (1-10\%), y rash cutáneo en el sitio de la inyección $(<1 \%)$. Los efectos adversos sistémicos observados fueron: fatiga, fiebre transitoria, cefalea, diarrea, tos, y disnea (1$10 \%$ ), así como náuseas, vómitos, mialgias, artralgias, 
somnolencia, mareos, anorexia $(<1 \%)$. Se recomienda el uso del AINE paracetamol ante la aparición de fiebre después de la vacunación. Las contraindicaciones de esta alternativa son: pacientes que recientemente hayan presentado COVID-19 y aún no se recuperen, individuos que hayan recibido plasma convaleciente previo a la vacunación (diferir 90 días la vacunación contra SARS-CoV-2) y pacientes con alergia conocida a algún componente de la vacuna o reacción alérgica grave a la dosis anterior. Se debe tener precaución en pacientes con trombocitopenia y trastornos de la coagulación, y epilepsia no controlada u otro trastorno neurológico progresivo $(6,7)$.

La OMS respecto a la vacuna Sinopharm es recomendada para edades de 18 años de edad y mayores, no se recomienda la vacunación de niños o adolescentes menores de 18 años de forma rutinaria, aunque los estudios están en curso (8).

Vacuna, es una suspensión de microorganismos vivos atenuados, inactivos, de una de sus partes o de un producto derivado de ellos que se administra para producir una infección similar a la infección natural, pero sin peligro para el que la recibe, con el objetivo de producir una respuesta inmunitaria que los proteja frente a ulteriores contactos con el germen del que se le ha vacunado. Si la protección se extiende a personas no vacunadas se habla de inmunidad de grupo o de rebaño $(9,10)$.

Anticuerpo (Inmunoglobulinas): son las defensas que desarrolla el sistema inmunológico del organismo para contrarrestar el agente causal de la enfermedad, ya sea por vía natural o por vacunación, eliminando las substancias externas al organismo (11).

Memoria inmunológica Capacidad que tiene el organismo de reconocer un antígeno con el que estuvo previamente en contacto y de responder de manera rápida y eficaz confiriendo protección al mismo, se lleva a cabo por los linfocitos B y T. Antígeno Cualquier substancia capaz de unirse específicamente a un anticuerpo o a un receptor de la célula $\mathrm{T}$ y generar una respuesta que ponga en marcha el sistema inmune (11).

\section{MATERIAL Y MÉTODOS}

El método general de la investigación es el científico, el método específico es el método descriptivo. El tipo de investigación estudio descriptivo y básico, el nivel de investigación es el descriptivo.
El diseño de la investigación es descriptivo simple.

El esquema es el siguiente:

O1 ----------- X ----------- O2

Donde $\mathrm{O} 1=$ Personal de Salud del

"Hospital El Carmen"

$\mathrm{X}=$ Vacuna Sinopharm

$\mathrm{O} 2=\quad$ Personal de Salud vacunado en el "Hospital

El Carmen"

La población estuvo conformado por 77 pacientes de la unidad de cuidados intensivos en un hospital nacional de la ciudad de Lima. Muestra censal conformada por los 77 pacientes de la unidad de cuidados intensivos de un hospital nacional de la ciudad de Lima, muestreo no probabilístico intencional

La población estuvo conformada por 1087 personales de Salud del hospital El Carmen.

Muestra conformada por los 284 personales de Salud del hospital El Carmen, determinados con la fórmula para la muestra probabilístico de aleatorio simple.

$\mathrm{Z}=1.96$

$\mathrm{N}=1087$

$\mathrm{P}=0.5$

$\mathrm{E}=0.05$

$$
n_{0}=\frac{Z^{2} N \cdot P \cdot Q}{Z^{2} P \cdot Q \cdot+(N-1) E^{2}}
$$

Criterios de inclusión:

Se recolectarán los datos directamente a cada personal de Salud post vacuna Sinopharm en ambas dosis, en el "Hospital El Carmen" Huancayo 2021, así recolectar los datos necesarios para la investigación, luego se realizará el estudio estadístico de las reacciones frecuentes anotadas y codificadas acorde a lo investigado. Los resultados se presentarán en tablas de frecuencia y figuras que denoten mejor los datos estadísticos, para luego realizar la discusión de los resultados obtenidos y llegar a las conclusiones necesarias. Finalmente se presentará el informe final.

\section{Criterios de exclusión:}

Se excluirá al personal que dejo en blanco algún dato del cuestionario.

Se excluirá al personal de salud que recibió la vacuna fuera del hospital El Carmen 
Se excluirá al personal de salud que recibió otro tipo de vacuna que no fuese el SINOPHARM.

La técnica de investigación observación, el instrumento ficha de observación, con una confiabilidad de 0,798 .

La técnica de investigación observación, se usó la encuesta y el instrumento fue un cuestionario, ambos aprobados por juicio de expertos.

\section{RESULTADOS}

En la tabla 1 y grafico 1 se observa que las reacciones más frecuentes post vacuna SINOPHARM en la primera y segunda dosis en el personal de Salud del Hospital El Carmen Huancayo 2021 fueron el dolor muscular con $16,6 \%$ en la primera dosis y con $13,5 \%$ en la segunda dosis, seguido de fatiga con $12,8 \%$ en la primera dosis y $2,9 \%$ en la segunda dosis, así también se tuvo a la fiebre con $6,4 \%$ y $8,7 \%$, luego

Tabla 1. Reacciones más frecuentes post vacuna SINOPHARM en la primera y segunda dosis en el personal de Salud del "Hospital El Carmen" Huancayo 2021

\begin{tabular}{|c|c|c|c|c|}
\hline \multirow[t]{2}{*}{ Reacciones } & \multicolumn{2}{|c|}{ 1ra dosis } & \multicolumn{2}{|c|}{ 2da dosis } \\
\hline & $\mathbf{n}$ & $\%$ & $\mathbf{n}$ & $\%$ \\
\hline Nasofaringitis & 0 & 0,4 & 10 & 4,8 \\
\hline Dolor De Estomago & 3 & 0,6 & 5 & 2,4 \\
\hline Estreñimiento & 5 & 0,9 & 0 & 0,0 \\
\hline Dificultad Para Tragar & 7 & 1,0 & 0 & 0,0 \\
\hline Picor De La Piel & 8 & 1,2 & 16 & 7,7 \\
\hline Hipersensibilidad & 9 & 1,3 & 3 & 1,4 \\
\hline Congestión Nasal & 10 & 1,3 & 6 & 2,9 \\
\hline Sequedad De La Garganta & 10 & 1,5 & 14 & 6,8 \\
\hline Tos & 12 & 1,5 & 10 & 4,8 \\
\hline Gripe & 12 & 1,7 & 0 & 0,0 \\
\hline Palpitaciones & 13 & 1,9 & 10 & 4,8 \\
\hline Dolor Orofaríngeo & 15 & 2,1 & 3 & 1,4 \\
\hline Goteo Nasal & 16 & 2,1 & 7 & 3,4 \\
\hline Estornudos & 16 & 2,2 & 3 & 1,4 \\
\hline Letargo & 17 & 2,3 & 3 & 1,4 \\
\hline Vómitos & 18 & 2,7 & 0 & 0,0 \\
\hline Pérdida De Apetito & 21 & 3,9 & 4 & 1,9 \\
\hline Dificultad Para Dormir & 30 & 4,0 & 3 & 1,4 \\
\hline Diarreas & 31 & 4,1 & 0 & 0,0 \\
\hline Dolor De Extremidades & 32 & 4,9 & 3 & 1,4 \\
\hline Somnolencia & 38 & 5,1 & 3 & 1,4 \\
\hline Mareos & 40 & 5,5 & 16 & 7,7 \\
\hline Dolor Articulaciones & 43 & 5,6 & 15 & 7,2 \\
\hline Nauseas & 44 & 6,4 & 0 & 0,0 \\
\hline Fiebre & 50 & 6,4 & 18 & 8,7 \\
\hline Fatiga & 50 & 12,8 & 6 & 2,9 \\
\hline Dolor Muscular & 100 & 16,6 & 28 & 13,5 \\
\hline Dolor & 129 & 0,4 & 21 & 10,1 \\
\hline
\end{tabular}

Fuente: cuestionario de recolección de datos 
los mareos con $5,5 \%$ y $7,7 \%$, dolor de articulaciones $5,6 \%$ y $7,2 \%$, dolor $0,4 \%$ y $10,1 \%$, picor de la piel $1,2 \%$ y $7,7 \%$, sequedad de la garganta $1,5 \%$ y $6,8 \%$, palpitaciones $1,9 \%$ y $4,8 \%$, somnolencia con $5,1 \%$ y $1,4 \%$, dolor de extremidades con $4,9 \%$ y $1,4 \%$, tos $1,5 \%$ y $4,8 \%$, goteo nasal $2,1 \%$ y $3,4 \%$, pérdida de apetito 3,9 y $1,9 \%$, dolor orofaríngeo $2,1 \%$ y $1,4 \%$, nasofaringitis $0,4 \%$ y $4,8 \%$, dificultad para dormir $4 \%$ y $1.4 \%$, congestión nasal $1,3 \%$ y $2,9 \%$, diarreas $4,1 \%$ y $0 \%$, letargo $2,3 \%$ y $1,4 \%$, y menos del $3,5 \%$ estornudos, gripe, hipersensibilidad, dificultad para tragar, estreñimiento, dolor de estómago.

\section{DISCUSIÓN}

Según los resultados mostrados en la tabla1, el dolor muscular con $16,6 \%$ en la primera dosis y con $13,5 \%$ en la segunda dosis, seguido de fatiga con $12,8 \%$ en la primera dosis y $2,9 \%$ en la segunda dosis, así también se tuvo a la fiebre con $6,4 \%$ y $8,7 \%$, luego los mareos con $5,5 \%$ y $7,7 \%$, dolor de articulaciones $5,6 \%$ y $7,2 \%$, dolor $0,4 \%$ y $10,1 \%$, picor de la piel $1 ., 2 \%$ y $7,7 \%$, sequedad de la garganta $1,5 \%$ y $6,8 \%$, palpitaciones $1,9 \%$ y $4,8 \%$, somnolencia con $5,1 \%$ $1,4 \%$, dolor de extremidades con $4,9 \%$ y $1,4 \%$, tos $1,5 \%$ y $4,8 \%$, goteo nasal $2,1 \%$ y $3,4 \%$, pérdida de apetito 3.9 y $1,9 \%$, dolor orofaringeo $2,1 \%$ y $1,4 \%$, nasofaringitis $0,4 \%$ y $4,8 \%$, dificultad para dormir $4 \%$ y $1,4 \%$, congestión nasal $1,3 \%$ y $2,9 \%$, diarreas $4,1 \%$ y $0 \%$, letargo $2,3 \%$ y $1,4 \%$, y menos del $3,5 \%$ estornudos, gripe, hipersensibilidad, dificultad para tragar, estreñimiento, dolor de estómago.

Se coincide con Tao Lina, experto en vacunas y responsable de la gestión de vacunas en el Centro de Shanghái para el Control y la Prevención de Enfermedades, afirma sobre SINOPHARM que tiene 73 efectos adversos son: reacciones locales: enrojecimiento, hinchazón, escleroma, sarpullido, picor en el lugar de la aplicación de la vacuna; y para las reacciones sistémicas: Dolor de cabeza, fiebre, fatiga, dolor muscular, dolor de articulaciones, tos, dificultad para respirar, náuseas, diarreas, picor de la piel, mareos, pérdida del apetito, vómitos, dolor orofaríngeo, dificultad para tragar, goteo nasal, estreñimiento, hipersensibilidad, letargo, somnolencia, dificultad para dormir, estornudos, nasofaringitis, congestión nasal, sequedad de la garganta, gripe, hipoestesia, dolor de extremidades, palpitaciones, dolor de estómago, sarpullido, anomalías de la piel, anomalía de las membranas mucosas, acné, dolor de ojos, molestias en los oídos, linfadenopatía, escalofríos, disgeusia (distorsión del sentido del gusto), pérdida del gusto, sensación anormal, temblores, dificultad para prestar atención, hemorragias nasales, asma, irritación de la garganta, amigdalitis, molestias en las extremidades, dolor de cuello, dolor de mandíbula, bultos en el cuello, úlceras en la boca, dolor de muelas, enfermedad esofágica, gastritis, decoloración de las heces, dolor de ojos, visión borrosa, irritación de los ojos, pérdida de visión (ceguera), dolor de oídos, nerviosismo, alta presión sanguínea, baja presión sanguínea, incontinencia urinaria, y retraso de la menstruación.

De igual manera Subirana (4) publicó en el diario el Comercio en octubre del 2021 según el MINSA la información detalla que de los 18 mil reportes de ESAVI notificados incluyen un total de 37,085 eventos adversos entre leves, moderados y graves, y corresponden a la vacuna contra la COVID-19 de los laboratorios Sinopharm, Pfizer, AstraZeneca y Johnson \& Johnson (aplicada fuera del país), señala que los ciudadanos inmunizados con la vacuna de Sinopharm presenta mayor cantidad de efectos secundarios con un total de 26346 eventos adversos, en tanto, los trastornos mayormente reportados fueron los del sistema nervioso, como cefalea y mareo; los trastornos generales y alteración en el lugar de administración, como el dolor en la zona de vacunación, malestar y pirexia; los trastornos gastrointestinales, como diarrea y náuseas; entre otros. Los más relevantes son cefalea $20,1 \%$, dolor en la zona de vacunación $12,4 \%$, mareo $5,4 \%$, malestar $5,2 \%$, Pirexia $5,1 \%$, Náuseas $4,7 \%$, Somnolencia $4,7 \%$, Fatiga $3,1 \%$, mialgia $2,8 \%$, diarrea $2,7 \%$, dolor en una extremidad 2,5\%, artralgia $1,9 \%$, dolor orofaríngeo $1,6 \%$, escalofríos $1,6 \%$, dolor de espalda $1,5 \%$, vómitos $1,1 \%$, parestesia $1,1 \%$, prurito $1 \%$, dolor torácico $0,9 \%$, tos $0,8 \%$, otros $19,8 \%$.

Por otro lado, se coincide con el estudio realizado por García (6), Vacunas contra la COVID-19. La propuesta de Sinopharm BBIBP-CorV, fue desarrollada en colaboración con el Beijing Institute of Biological Products quien indica que los efectos adversos fueron de gravedad leve o moderada y se desarrollaron durante los primeros siete días después de la inoculación. Los efectos adversos locales fueron los siguientes: dolor en el sitio de la inyección (> $10 \%$ ); eritema, hiperemia, prurito, edema o hinchazón en el sitio de la inyección (1-10\%), y rash cutáneo en el sitio de la inyección $(<1 \%)$. Los efectos adversos sistémicos observados fueron: fatiga, fiebre transitoria, cefalea, diarrea, tos, y disnea (1-10\%), así como náuseas, vómitos, mialgias, artralgias, somnolencia, mareos, anorexia $(<1 \%)$. 
A diferencia se tiene a Gironzini (5), quien concluye que la reacción adversa más frecuente fue el dolor en el sitio de inoculación, con una frecuencia de $42.1 \%$ y $43.2 \%$ en la primera y segunda dosis, las reacciones sistémicas más frecuentes fueron cefalea y astenia. No se reportaron reacciones adversas graves.

Según la OMS refiere que las reacciones adversas de la vacuna Sinopharm fueron en su mayoría de leves a moderados y de corta duración, como reacciones locales muy frecuentes $(\geq 1 / 10)$ : Dolor en el lugar de la inyección, poco frecuentes $(\geq 1 / 1000$ a $<1 / 100)$ : Enrojecimiento, hinchazón, induración, picazón y dentro los sistémicos: Muy frecuentes $(\geq 1 / 10)$ : Dolor de cabeza, frecuentes $(\geq 1 / 100$ a $<1 / 10)$ :Fiebre, fatiga, mialgia, artralgia, tos, disnea, náuseas, diarrea, prurito. Poco frecuentes $(\geq 1 / 1000 \mathrm{a}<1 / 100)$ : Mareos, anorexia, dolor orofaríngeo, disfagia, congestión nasal, estreñimiento, hipersensibilidad. Raros $(\geq 1 / 10$ 000 a <1/1 000): Letargo, somnolencia, dificultad para conciliar el sueño, estornudos, nasofaringitis, congestión nasal, garganta seca, gripe, hipoestesia, dolor en las extremidades, palpitaciones, dolor abdominal, erupción, mucosa cutánea anormal, acné, oftalmodinia, molestias en el oído, linfadenopatía. Muy raros $(<1 / 10$ 000): Escalofríos, disfunción del gusto, pérdida del gusto, parestesia, temblor, trastorno de atención, epistaxis, asma, irritación de garganta, amigdalitis, malestar físico, dolor de cuello, dolor de mandíbula, bulto en el cuello, úlceras en la boca, dolor de muelas, trastornos del esófago, gastritis, decoloración fecal, oftalmodinia, visión borrosa, irritación ocular, dolor de oído, tensión, hipertensión, hipotensión, incontinencia urinaria, menstruación retrasada y no se conoce cuadros de anafilaxia.

Para el primer objetivo específico: Detallar la edad del personal de salud que recibieron la primera y segunda dosis de la vacuna contra la Covid-19 Sinopharm en el Hospital El Carmen, Huancayo 2021, se tiene entre 20 a 49 años en un $71,1 \%$, seguido de 50 a 65 años con $28,9 \%$ y no se tiene ningún personal mayor de 65 años de edad, se coincide con Gironzini (5), quien determina la edad media fue de 26,61 años; mencionando que no se tiene más estudios para citarlos como antecedentes. Según la base teórica la OMS da a conocer respecto a la vacuna Sinopharm es recomendada para edades de 18 años de edad y mayores, no se recomienda la vacunación de niños o adolescentes menores de 18 años de forma rutinaria, aunque los estudios están en curso
Para el segundo objetivo específico: Detallar el sexo del personal de salud que recibieron la primera y segunda dosis de la vacuna contra la Covid-19 Sinopharm en el Hospital El Carmen, Huancayo 2021 el 80,3\% corresponde al sexo femenino y el $19,7 \%$ corresponde al sexo masculino se coincide con Gironzini (5), quien demostró que la muestra estaba conformada por 35 hombres $(36,8 \%)$ y 60 mujeres $(63,2 \%)$. No se cuenta con más estudios ni bibliografía donde señale que la vacuna es sólo para un determinado género.

Para el tercer objetivo específico: Detallar las reacciones post vacuna del personal de salud que recibieron la primera y segunda dosis de la vacuna contra la Covid-19 Sinopharm en el Hospital El Carmen, Huancayo 2021, el 66,6\% no reaccionaron en la primera dosis así también el 52,4\% no reaccionaron en la segunda dosis, pero el $33.5 \%$ sí reaccionaron en la primera dosis y el $48.9 \%$ en la segunda dosis, caso similar se tiene encontraron García et al., (6). La propuesta de Sinopharm BBIBP-CorV fue desarrollada en colaboración con el Beijing Institute of Biological Products quien señala que el efecto adversó se observó después de los siete días post vacunados y en porcentajes menores al $10 \%$ a diferencia de Gironzini (5), quien demostró que $72(75,8 \%)$ internos de medicina refirieron haber presentado alguna reacción adversa inmediata. $60(63,2 \%)$ participantes manifestaron reacciones adversas inmediatas en la primera dosis mientras que un total de $52(54,7 \%)$ participantes las presentaron en la segunda dosis.

Para el cuarto objetivo específico: Registrar si el personal de salud fue positivo a Covid 19 antes o después de ser vacunado con la primera o segunda dosis con la vacuna contra la Covid-19 Sinopharm en el Hospital El Carmen, Huancayo 2021, el 59,2\% salió positivo a Covid 19 antes de ser vacunado y el $18,3 \%$ dio positivo después de ser vacunado contra la covid 19. Para éste objetivo no se cuenta con antecedentes para discutir por ser un tema inédito y nuevo a nivel nacional y mundial. Según la literatura En relación a la vacunación antes, durante y después de la vacunación, todas las personas deben seguir cumpliendo las directrices en vigor para la protección frente a la COVID 19 en su región (uso de mascarilla, distancia física, higiene de manos). Según los datos actuales y dado el suministro limitado de vacunas, la reinfección sintomática es poco común dentro de los 6 meses posteriores a una infección natural inicial, las personas con infección por SARS CoV 2 confirmada 
mediante PCR en los últimos 6 meses pueden optar por retrasar la vacunación hasta cerca del final de este periodo.

Para el objetivo quinto: Registrar alguna reacción inmediata del personal de salud que recibieron la primera y segunda dosis de la vacuna contra la Covid-19 Sinopharm en el Hospital El Carmen, Huancayo 2021. En la primera dosis el 39,4\% no tuvieron ninguna reacción, el $18,3 \%$ tuvieron reacciones locales, el 16,2\% hinchazón, 16,2\% picor en la zona de vacunación, el 4,9\% escleroderma, el $4,9 \%$ salpullido; en la segunda dosis el $62,7 \%$ no tuvieron ninguna reacción, el 10,9\% hinchazón, el $9,5 \%$ picor en la zona de vacunación, el $7 \%$ reacciones locales, el $7 \%$ salpullido y el $2,8 \%$ escleroderma. Se difiere Gironzini (5), con $60(63,2 \%)$ participantes manifestaron reacciones adversas inmediatas en la primera dosis mientras que un total de $52(54,7 \%)$ participantes las presentaron en la segunda dosis. No se tiene otros estudios para la discusión así también la literatura menciona de reacciones adversas $\sin$ mencionar si es inmediato o mediato.

Para el objetivo específico sexto: Detallar las reacciones posteriores del personal de salud que recibieron la primera y segunda dosis de la vacuna contra la Covid-19 Sinopharm en el Hospital El Carmen, Huancayo 2021, no reaccionaron en la primera dosis el $54,5 \%$ y $90,1 \%$ en la segunda dosis y sí presentaron reacciones el $45.4 \%$ en la primera dosis y $9,9 \%$ en la segunda dosis. No se cuenta con antecedentes para la discusión respecto a éste punto.

\section{Correspondencia:}

Jenny Giovanna Poma Salinas

Correo electrónico: gpoma@uroosevelt.edu.pe

\section{REFERENCIAS BIBLIOGRAFICAS}

1 Alvarez, J, García, C. Vacunas para SARS-COv-2: entre la urgencia y la ciencia. Observatorio de Salud. Mendoza: Universidad Juan Agustín Maza; 2020.

2. Organización Mundial de la Salud. Vacunas e inmunización: ¿qué es la vacunación? Ginebra: Organización Mundial de la Salud; 2019. (citado el 30 de diciembre 2020). Disponible: https://www. who.int/es/news-room/q-a-detail/vaccines-andimmunization-what-is-vaccination?adgroupsurvey $=\{$ adgroupsurvey $\&$ gclid $=\mathrm{Cj} 0 \mathrm{KCQ}$ iAyoe $\mathrm{CBh}$ CTARIsAOfpKxjA7nPWT2CoH11XSEZGC9MKAJ Q3zPR82wndK0YSQ6vX3EaGE6j1I5gaAirKE ALw_wcB
3. La ética formal Kantiana. En: Webdianoia.La Filosofia de Kant. (Citado el 30 de diciembre 2020). Disponible en: https://www.webdianoia.com/ moderna/kant/kant_fil_etica.htm

4. Subirana K. COVID-19: ¿Cuáles son los efectos secundarios de las vacunas reportados en Perú? Lima: Diario El Comercio; 2021.

5. Gironzini P. Reacciones adversas inmediatas a la vacuna inactivada contra el Sars Cov-2 BBIBP-CORV en 95 internos de medicina del hospital III Goyeneche - MINSA, Arequipa 2021. Tesis de título profesional. Arequipa: Universidad Nacional San Agustin; 2021. (Citado el30 de diciembre2020). Disponible en: http:// repositorio.unsa.edu.pe/handle/20.500.12773/12580

6. García Z Peron L, Ramirez I, et al. Vacunas contra la COVID-19. Acta Med. 2021;19(3):429-444. Doi:10.35366/101742.

7. Eduardo, M. Conceptos importantes sobre inmunizaciones. Acta méd. Peruana. 2007; 24(1): 59-64. (Citado el 30 de diciembre 2020). Disponible en: http://www.scielo.org.pe/scielo.php?script=sci arttext\&pid=S1728-59172007000100013

8. Organización Mundial de la Salud. V a c u n a contra la COVID-19 (Vero Cell), Inactivada (Sinopharm). Beijing Institute of Biological Products Co., Ltd. Ginebra: Organización Mundial de la Salud; Mayo 2021.

9. Ministerio de salud Argentina. Manual del Vacunador Vacuna SINOPHARM. Buenos Aires: Ministerio de salud Argentina; febrero 2021. (Citado el 30 de junio 2020). Disponible en: https://bancos.salud. gob.ar/sites/default/files/2021-03/manual-vacunadorsinopharm_12-3-2021.pdf

10. Martínez-Mateo P, Bustos-Fonseca M, Gil-Díaz M. Actualización en vacunas. Teoría, realidades y mitos. Medicina de Familia. SEMERGEN. 2012; 38 (3):160166.

11. Benemérita Universidad Autónoma de Puebla. Norma PAI. Conceptos y Principios generales de Inmunización. Puebla: Benemérita Universidad Autónoma de Puebla; 2020. (Consultado 21 de marzo 2021)Disponible en: https://www.studocu.com/es$\mathrm{mx} /$ document/benemerita-universidad-autonomade-puebla/inmunologia/apuntes/conceptos-yprincipios-generales-de-inmunizacion/6149435/ view.

Recibido: $12 / 09 / 2021$

Aceptado: 17/12/2021 